\title{
2.4 Sozialwissenschaften
}

\section{Siegfried Geyer, Thomas Abel}

Anders als in der Medizin werden die für Forschung und Praxis nötigen Daten im Bereich der Sozialwissenschaften in erster Linie über Fragebogen und nicht durch die Messung biologisch-medizinischer Parameter gewonnen. In der Gesundheitsförderung und der Prävention setzt man Fragebogen häufig dann ein, wenn man etwas über das Wissen, die Wahrnehmungen oder subjektiven Beurteilungen zu bestimmten Verhaltensweisen, Zuständen oder Bedürfnissen von Personen bzw. Personengruppen erfahren möchte. Solche systematischen Befragungen, die das Ziel haben, Daten zu einem bestimmten Thema zu erheben, nennt man auch Surveys. Kenntnisse in der Entwicklung und Anwendung von Fragebogen sind unentbehrlich, wenn es darum geht, Public-Health-Studien zu beurteilen oder gar selbst durchzuführen.

In diesem Abschnitt beschäftigen wir uns zuerst mit der Formulierung von guten Fragen und möglichen Antworten. Anschließend betrachten wir unterschiedliche Methoden der Datenerhebung und diskutieren ihre Vor- und Nachteile. Dabei gehen wir auch kurz auf die Methodik der qualitativen Verfahren ein.

Schweizerische Lernziele: CPH 8, CPH 20

\subsubsection{Was ist eine gute Frage?}

Eine klare und verständliche Formulierung der Fragen ist die wichtigste Voraussetzung dafür, dass sich aus den mit Hilfe von Fragebogen erhobenen Daten später durch Interpretation auch Schlüsse ziehen lassen. Hierzu müssen Forscher und Befragte eine gestellte Frage in gleicher Weise verstehen und interpretieren können. Dies ist in der Praxis keineswegs selbstverständlich. Denn nicht immer sprechen die Konstrukteure eines Fragebogens und die Adressaten, an die sich der Fragebogen richten soll, im Hinblick auf den Wortschatz und das sprachliche Niveau die gleiche Sprache. Wenn eine Frage verstanden wurde, dann müssen die Befragten auch über die notwendige Information verfügen, sie zu beantworten. Dazu gehört nicht nur das Wissen um eine Antwort, sondern auch genügend Zeit, um sich an die Information zu erinnern und die Antwort dann zu formulieren.

In Lebensqualitätsfragebogen wird z. B. danach gefragt, wie häufig bestimmte Symptome innerhalb eines definierten Zeitraums aufgetreten sind. Da jedoch Ereignisse, die als wenig relevant erachtet wurden, aufgrund der Struktur des menschlichen Gedächtnisses nach einer gewissen Zeit vergessen werden, sind die Antworten hierauf unter Umständen wenig präzise. Seltene Ereignisse, wie etwa die Häufigkeit des Auftretens von Symptomen oder die Zahl von Arztbesuchen, werden in der Regel gezählt. Bei häufigeren Ereignissen basieren die Angaben dagegen auf groben Schätzungen und sind entsprechend ungenau.

Antworten werden in der Regel durch solche Sachverhalte bestimmt, die zum Zeitpunkt der Fragestellung im Gedächtnis der Befragten präsent sind. Ist ein längerer Erinnerungsprozess erforderlich, muss den Befragten genügend Zeit zur Verfügung stehen. Jedoch auch dann können im Ergebnis erhebliche Urteilsfehler auftreten. So kann z. B. 
die Zahl der Arztbesuche falsch eingeschätzt werden, wenn sich die Befragten an ein bestimmtes Datum nicht direkt erinnern können. Oft wird es dann aus anderen Ereignissen rekonstruiert. Bei dieser Rekonstruktion können jedoch Irrtümer vorkommen. Schließlich können Fragen, die den Befragten peinlich oder in anderer Weise unangenehm sind, zu einer Antwortverweigerung führen. Beispiele hierfür sind Fragen nach dem Alkoholkonsum, nach Sexualpraktiken oder auch nach dem Einkommen.

Die Qualität der gegebenen Antworten ist jedoch nicht nur von der Verständlichkeit der Fragen abhängig, sondern auch von der Länge des Fragebogens. Mit zunehmender Befragungsdauer nehmen Konzentrationsprobleme bei den Befragten zu, das Risiko von Urteilsfehlern steigt, während die Motivation zur Teilnahme sinkt. Dies ist insbesondere bei alten Menschen und Menschen mit Erkrankungen zu berücksichtigen.

Bei der Konstruktion eines Fragebogens ist die Entscheidung, ob die Antwortmöglichkeiten vorgegeben (sog. geschlossene Fragen) oder die Antworten offen gelassen werden (sog. offene Fragen), vom Verwendungszweck und der geplanten Vorgehensweise bei der Auswertung abhängig. Fragen mit vorgegebenen Antwortmöglichkeiten sind in der Regel schneller zu beantworten, die quantitativen Informationen sind leichter auszuwerten. Geschlossene Fragen grenzen jedoch den Antworthorizont der Befragten auf die vorgegebenen Alternativen ein, und zwar auch dann, wenn die zusätzliche Option einer offenen Antwort vorgegeben wird. Die Antwortvorgaben bei geschlossenen Fragen sollten immer einen möglichst hohen Grad an Eindeutigkeit haben.

Beispiel für eine geschlossene Frage:

Wie häufig haben Sie in den letzten sechs Monaten wegen einer Erkrankung oder wegen Beschwerden eine Arztpraxis aufgesucht?

Antwortmöglichkeiten:

$\square$ Gar nicht $\quad \square$ einmal $\quad \square$ zwei- bis viermal $\quad \square$ mehr als viermal

Wenn über den Gegenstand einer Frage wenig bekannt ist, sollten die Antworten offen gelassen werden. Die Antworten auf solche offenen Fragen sind meist subjektive Einschätzungen der Befragten, in die eine möglichst große Bandbreite an Informationen einfließen sollte. Offene Fragen liefern v. a. qualitative Informationen. Ihre Auswertung ist meist aufwendig.

Beispiel für eine offene Frage:

Gibt es Ihrer Meinung nach Zusammenhänge zwischen Ihrer Arbeitslosigkeit und Ihrem Gesundheitszustand? Und wenn ja, welche?

Antwort:

(Bitte verwenden Sie so viele Zeilen, wie Sie möchten.)

\subsubsection{Was führt zu einer guten Antwort?}

Bei der Konstruktion von Fragebogen kann man auf mehrere Antwortformat-Optionen zurückgreifen. Je nach Verwendungszweck können sie innerhalb eines Fragebogens 
auch miteinander kombiniert werden. Die Web-Abb. 2.4.1 auf unserer LehrbuchHomepage zeigt einen Abschnitt aus einem Fragebogen der Eidgenössischen Jugendbefragung CH-X 2010 - Vertiefungsfragen zur Gesundheit, bei dem verschiedene Antwortformat-Optionen verwendet wurden.

\section{Ratingskalen/Ordinalskalen}

Am häufigsten werden Ratingskalen verwendete, die mehrere Antwortalternativen anbieten und den Befragten dadurch eine abgestufte Antwort ermöglichen. Dabei sollten die Alternativen so formuliert werden, dass sich die einzelnen Kategorien auf den gleichen Inhalt beziehen und semantisch die gleichen Abstände haben. Diese semantische Äquidistanz wurde bisher für drei Beurteilungsdimensionen untersucht:

- Häufigkeit: nie - selten - gelegentlich - oft - immer

- Intensität: nicht - wenig - mittelmäßig - ziemlich - sehr

- Bewertung von Aussagen: stimmt nicht - stimmt wenig - stimmt mittelmäßig - stimmt ziemlich - stimmt sehr

Werden solche verbalen Quantifizierer verwendet, muss darauf geachtet werden, dass die Begriffe in der Wahrnehmung der Befragten etwa gleiche Abstände haben. Die Zahl der Stufen ist dabei jedoch nicht festgelegt. In der Regel werden zwischen fünf und sieben Antwortalternativen gewählt. Hierbei gilt es immer, zwischen den Differenzierungserfordernissen des zu beurteilenden Gegenstands und den Fähigkeiten der Befragten einen praktikablen Kompromiss zu finden.

\section{Kategorialskalen}

Eine solche Skala besteht aus sich gegenseitig ausschließenden Kategorien, die qualitativer Art und ohne eine natürliche Ordnung sind. Ein Beispiel hierfür ist die Klassifizierung von Personen nach ihrem Familienstand (schweizerisch: Zivilstand) in die Kategorien "ledig", "verheiratet", "geschieden" oder „verwitwet". Die Befragten können dort in Abhängigkeit von der Instruktion entweder nur eine oder auch mehrere Antworten ankreuzen. Mehrere Antworten könnten z. B. auch bei einer Frage nach vorhandenen Stress-Symptomen ausgewählt werden. In anderen Fällen können die Befragten aufgefordert werden, Begriffe in eine Rangreihe zu bringen.

\section{Die Testung von Fragebogeninstrumenten}

Es ist nun keineswegs sicher, dass ein Fragebogen in der Form, wie er entwickelt wurde, ohne weiteres auch später in der Praxis verwendet werden kann. Da Surveyfragen meist von Fachleuten entworfen werden, muss die verwendete Sprache nicht mit der der Zielgruppe übereinstimmen. In der praktischen Anwendung kann es zu Problemen kommen, wenn die Befragten eine Frage anders verstehen als von den Fragebogenkonstrukteuren gedacht. Auch können die verwendeten Begriffe mehrdeutig sein und dann von Befragten und Fragebogenkonstrukteuren unterschiedlich verstanden werden. Beides kann später zu erheblichen Schwierigkeiten in der Interpretation der gewonnenen Daten führen. Darüber hinaus können abstrakte Begriffe in ihrem inhaltlichen Verständ- 
nis divergieren. So kann z. B. eine Frage nach dem schweizerischen Gesundheitssystem so beantwortet werden, dass Befragte, die im Versicherungswesen arbeiten, bei ihrer Beantwortung primär das Versicherungssystem im Blick haben. ÄrztInnen denken dagegen in erster Linie an die ärztliche Versorgung. Patienten beantworten die Frage vor dem Hintergrund ihrer eigenen Erfahrung mit ÄrztInnen bzw. Einrichtungen der medizinischen Versorgung.

Bei der Lösung der daraus resultierenden Probleme können routinemäßig angewandte Standardpretests eine Hilfe sein. Hierbei werden die entwickelten Fragebogen in Interviews unter möglichst realistischen Befragungsbedingungen getestet. Die Interviewer registrieren dort die von den Befragten unaufgefordert abgegebenen Kommentare und melden diese an die Studienleitung zurück. Das Verfahren kann nur grobe Fehler aufdecken. Antworten von Befragten, die irrtümlich der Überzeugung sind, dass sie eine Frage korrekt verstanden haben, bleiben ungeprüft als richtig stehen. Nach dem derzeitigen Wissensstand können Standardpretests zur Schätzung des für ein Interview notwendigen Zeitaufwands dienen, nicht jedoch zur Aufdeckung von solch spezifischen Verständnisproblemen. Den Fragebogenkonstrukteuren steht mittlerweile ein umfangreiches Instrumentarium zur Testung der Verständlichkeit von Surveyfragen zur Verfügung. Nach dem derzeitigen Stand der Methodenforschung muss der Einsatz eines nicht getesteten Fragebogens als Fehler gewertet werden. Das am häufigsten verwendete Testverfahren ist das Probing. Hierbei werden potentiell unklare Begriffe oder auch eine ganze Frage auf ihre Verständlichkeit hin untersucht. Bislang gibt es noch keine komplette Liste von standardisierten Regeln zur Überprüfung von Fragebogen. Auch die angemessene Fallzahl für einen Pretest ist nicht festgelegt. Wenn jedoch komplexere Inhalte abgefragt werden und/oder die Grundgesamtheit der Befragten heterogen ist, werden größere Fallzahlen (ca. 20 Fälle) als angemessen erachtet.

\subsubsection{Quantitative Methoden zur Erhebung von Daten}

\section{Persönliche Befragung}

Die klassische Form der Befragung ist das persönliche Interview. Aus Kostengründen wird mittlerweile jedoch die Mehrzahl der Befragungsstudien mit Hilfe anderer Methoden durchgeführt. Bei der persönlichen Befragung sitzen sich Befragte/r und Interviewer/in gegenüber. Normalerweise verliest der/die Interviewer/in die Fragen, und die darauf gegebenen Antworten des/der Befragten werden registriert. Es ist auch möglich, Antwortalternativen in Form von Karten vorzulegen. Darüber hinaus können wahlweise Abbildungen, Modelle oder Fragebogen zum Selbstausfüllen eingesetzt werden.

Werden bei persönlichen Interviews Papierfragebogen eingesetzt, dann müssen die auf diese Weise gewonnenen Informationen anschließend in eine elektronische Form gebracht werden. Durch den Einsatz von Computern direkt bei der Befragung ist dies heute meist nicht mehr nötig. Solche "Computer-Assisted Personal Interviews" (CAPI) ermöglichen es, Kontrollen in die Dateneingabe einzubauen und nötige Korrekturen unmittelbar vornehmen zu lassen. Dabei wird durch ein Hintergrundprogramm automatisch geprüft, ob ein eingegebener Wert innerhalb eines definierten Bereichs liegt. Nach dem Abschluss der Befragung liegt dann bereits ein auswertungsfähiger Datensatz vor.

Durch die persönliche Form der Kommunikation kommt den Interviewern bei dieser Form der Datenerhebung eine besondere Rolle zu. Sie müssen von den Befragten ak- 
zeptiert werden und - in Abhängigkeit von der Studienthematik - auch in der Lage sein, ein gewisses Vertrauensverhältnis aufzubauen. Hierzu ist eine gründliche Schulung der Interviewerlnnen notwendig. Lange Zeit lernte man in solchen Schulungen, dass das Interviewerverhalten eher distanziert und auf die alleinige Gewinnung von Informationen ausgerichtet sein sollte. Untersuchungen haben jedoch gezeigt, dass diese Form von den Befragten oft als kalt und teilnahmslos empfunden wird. Eine emotional warme und unterstützende Form der Befragung erzielt bei inhaltlich neutraler Gesprächsführung deutlich bessere Daten. InterviewerInnen sollten dabei über ein ausreichendes Selbstbewusstsein verfügen und in der Lage sein, potentielle Befragte zu einer Teilnahme zu animieren. Darüber hinaus müssen sie fähig sein, sich unterschiedlichen Situationen flexibel anzupassen. Die Bedeutung dieser Eigenschaften steigt mit der Komplexität einer Studie.

\section{Telefonische Befragung}

Mit zunehmender Telefondichte wurde die Möglichkeit, die Datenerhebung bei Studien über das Telefon durchzuführen, immer häufiger genutzt. Telefoninterviews erfordern bei kleineren Stichproben keine großen infrastrukturellen Voraussetzungen und können relativ kostengünstig durchgeführt werden. Wenn die Telefondichte in einer Bevölkerung hoch genug ist, besteht darüber hinaus die Möglichkeiten, daraus große und/oder repräsentative Stichproben zu ziehen, sodass das Telefon sowohl für umfangreichere Surveys als auch für kleinere Erhebungen genutzt werden kann. Etwa seit der Jahrtausendwende sinkt die Zahl der Festnetzanschlüsse in den meisten westlichen Industrienationen jedoch zugunsten der Mobiltelefone kontinuierlich ab, sodass eine Repräsentativität von Telefonbefragungen über Festnetzanschlüsse schwieriger zu erzielen ist. Darüber hinaus sind die Verweigerungsraten bei Telefonbefragungen sehr hoch, da sich Telefonbesitzer durch die immer häufigeren Umfragen und Werbeanrufe belästigt fühlten. Nach einer Übersicht aus den USA sanken die Antwortraten bei Surveys mit kurzer Laufzeit (5 Tage) zwischen 1997 und 2003 von 36\% auf 25\%, bei aufwändigeren Studiendesigns mit mehrfacher Kontaktaufnahme von $61 \%$ auf $50 \%$.

Aus Sicht der Untersucher sind telefonische Befragungen von Vorteil, da hier im Vergleich zu persönlichen Befragungen die Wege- und Reisekosten wegfallen. Dadurch können in einer bestimmten Zeiteinheit wesentlich mehr Interviews durchgeführt werden. In größeren Studien kann der Einsatz von InterviewerInnen zentral über ein Surveylabor organisiert werden. Oftmals werden die Interviews dann als Computer-Assisted Telephone Interviews (CATI) durchgeführt. Hierdurch sind eine bessere Kontrolle der Studiendurchführung sowie eine bessere Supervision seitens der Interviewerlnnen möglich. Ein weiterer Vorteil ist, dass Befragte bei Umzügen nicht mehr aus der Stichprobe ausscheiden, sofern sie ihre Telefonnummer beibehalten.

Andererseits muss das Studiendesign bei Telefonsurveys dem Medium angepasst werden. Zur Übermittlung von Informationen steht hier - zumindest bis heute - nur das gesprochene Wort zur Verfügung. Fragebogen, die für ein persönliches oder für ein schriftliches Interview konzipiert wurden, können daher für die telefonische Befragung untauglich sein. Sie sind möglicherweise zu komplex oder verwenden optische Präsentationen, wie z. B. Bilder oder Tabellen. In diesen Fällen muss eine Vereinfachung bzw. Adaptation des Fragebogens vorgenommen werden. Wegen der begrenzten Gedächtnisspanne der telefonisch Befragten ist eine Präsentation von Antwortskalen oder länge- 
ren Listenfragen nicht möglich. Alternativ hierzu müssen Fragen zerlegt und die vorgegebenen Antworten in kategoriale bzw. in Ja/ Nein-Formate transformiert werden. Da es bei reinen Telefoninterviews nicht möglich ist, zusätzliches Stimulusmaterial wie Fotos, Karten oder visuelle Hilfen zu verwenden, kann alternativ ein zweistufiges Verfahren gewählt werden. Hierbei wird zunächst der Kontakt zu den Befragten aufgebaut und erst nach der Zusendung dieses Materials dann das eigentliche Telefoninterview durchgeführt.

Bei Telefoninterviews ist die Latenzzeit zwischen Frage und Antwort kürzer als bei anderen Befragungsformen. Bei komplexeren Inhalten sowie bei Fragen, bei denen die Befragten auf ihre Erinnerungen zurückgreifen müssen, kann das zu einer vergleichsweise niedrigen Zuverlässigkeit (Reliabilität) führen. Auch ist die Art des Kontakts am Telefon anonymer als bei einer persönlichen Befragung. Bei sensiblen Themen (wie z. B. beim Thema „häusliche Gewalt") kann ein höherer Grad an Anonymität eine Befragung erst möglich machen.

\section{Schriftliche Befragung}

Bei schriftlichen Befragungen werden die Fragebogen per Post verschickt oder auf eine andere Art ausgeteilt. Dabei muss darauf geachtet werden, dass kein direkter Kontakt zwischen Forschern und Befragten während des Ausfüllens besteht. Die Rücklaufquoten können stark zwischen 10\% bis $90 \%$ schwanken. Dies ist u. a. durch unterschiedliche Merkmale der Zielgruppen erklärbar. So sind Bevölkerungssurveys, die ohne ein offensichtliches Schwerpunktthema durchgeführt werden, anfälliger für eine geringe Rücklaufquote als thematisch enger definierte Befragungen. Auch bei bestimmten Bevölkerungsgruppen (u.a. bei Menschen mit hohem Zeitdruck, wie etwa Personen, die Beruf und Familie miteinander vereinbaren müssen) muss mit niedrigeren Beteiligungen gerechnet werden. Hohe Rücklaufquoten von über $70 \%$ können v. a. dann erreicht werden, wenn bei den Befragten eine hohe persönliche Betroffenheit vorliegt (z. B. bei Patientlnnen), wenn sie sich von der Teilnahme einen positiven Nutzen versprechen oder wenn ihnen die durchführende Institution bekannt ist.

Ein Programm zur Steigerung des Rücklaufs bei schriftlichen Befragungen („The Taylored Design Method [TDM]" von Dillman et al.) beinhaltet die folgenden Maßnahmen:

- Fragebogen: Der Fragebogen sollte als gebundenes, ansprechend gestaltetes Heft konstruiert werden. Er sollte mit einem interessanten, aber neutral gestalteten Umschlag aus festerem Papier versehen sein. Die optimale Länge eines Fragebogens wird in der Literatur mit 12 bis 16 Seiten angegeben.

- Anreize: Die Befragten sollten eine kleine Anerkennung (keine Bezahlung!) für das Ausfüllen des Fragebogens erhalten. Verschiedene Studien konnten zeigen, dass dadurch auch die Bereitschaft zur Teilnahme an Wiederholungsbefragungen steigt.

- Mehrfache Kontaktaufnahme: Um die Rücklaufquoten zu erhöhen, sollten wenn nötig insgesamt vier Kontaktaufnahmen vorgesehen werden (erste Versendung und drei Erinnerungen).

- Frankierter Rückumschlag: Um die Bearbeitung des Fragebogens für die Befragten so einfach wie möglich zu machen, sollte jeweils ein frankierter Rückumschlag beigelegt werden. 
- Anerkannte Autorität: Dem Fragebogen sollte neben einem personalisierten Anschreiben ein unterstützender Begleitbrief einer anerkannten Autorität beiliegen. Diese Persönlichkeit sollte im Hinblick auf ihre soziale Anerkennung in der Gruppe der Befragten sorgfältig ausgewählt werden und einen Bezug zur Thematik der Studie haben.

\section{Internetbefragungen}

Aufgrund der zunehmenden Verbreitung des Internets wird dieses Medium immer häufiger auch zu Befragungen, etwa für internetbasierte Gesundheitssurveys, genutzt. Onlinebefragungen erleichtern es, komplexere Fragenabfolgen in Surveys einzubinden, ohne dass hier ein größeres Fehlerrisiko besteht. So kann z.B. die auf dem Bildschirm erscheinende Abfolge der Fragen automatisch geändert werden, wenn die nächste Frage von der Antwort der vorhergehenden abhängt. Darüber hinaus können Bilder, Filme und andere Medien flexibel eingesetzt werden.

Die Verwendung von Onlinebefragungen hat in den letzten Jahren deutlich zugenommen. Bisher (2011) gibt es allerdings nur wenige Informationen zur Qualität der auf diese Weise gewonnenen Daten. Derzeit unterscheiden sich Internetnutzer und Nichtnutzer noch durch das Alter. Auch gibt es bei vorhandenem Internetzugang Unterschiede in der Vertrautheit der Nutzer mit dem Medium. Dies hat Auswirkungen auf die Erreichbarkeit von Zielgruppen und damit auch auf die Repräsentativität der so gewonnenen Daten. Im Vergleich zu telefonisch gesammelten Daten zeigte sich jedoch, dass die Internetbefragungen genauere Eigenbeschreibungen der Befragten erbrachten. Darüber hinaus ergab sich auch eine höhere Reliabilität und Validität (s. Kap. 2.1.4) der Daten. Das Wissen über die Möglichkeiten und Grenzen von Internetsurveys ist zum gegenwärtigen Zeitpunkt jedoch noch lückenhaft.

\subsubsection{Qualitative Datenerhebungsverfahren}

Mit Hilfe der bisher beschriebenen standardisierten Verfahren werden quantitative Daten gewonnen. Es ist jedoch auch möglich, qualitative Verfahren zur Datengewinnung einzusetzen. In Public Health kommen diese Verfahren z. B. zur Anwendung, wenn es gilt, verständliche Fragen für Fragebogen zu entwickeln und diese dann mit Hilfe von Fokusgruppen (s.u.) zu testen. Qualitative Verfahren können auch dazu dienen, die in quantitativen Befragungen erzielten Erkenntnisse durch detailliertere Informationen zu ergänzen (z. B. mit Hilfe von episodischen oder fokussierten Interviews bzw. Fallstudien, s. u.). Wegen des erheblich größeren Zeit- und Personalaufwands bei der Erhebung und Auswertung dieser Daten können solche Methoden jedoch jeweils nur bei relativ kleinen Fallzahlen eingesetzt werden. Am häufigsten werden die folgenden qualitativen Verfahren angewandt:

\section{Narrative Interviews}

Die Befragten werden hierbei zuerst über die Modalitäten des Vorgehens informiert. Anschließend werden sie aufgefordert, über ein zuvor festgelegtes Thema zu berichten. Ein solches Thema kann ein vergangenes Erlebnis sein, wie z. B. die eigene Krankengeschichte, die dann sowohl beschrieben als auch bewertet werden soll. Die Länge 
der Erzählphase wird durch die Befragten selbst bestimmt. Kommentierungen oder Nachfragen von Seiten der Interviewer sollen weitgehend unterbleiben.

\section{Episodische Interviews}

In episodischen Interviews werden die Befragten aufgefordert, über spezifische Situationen (z.B.: „In welcher Situation sind Sie sich zum ersten Mal ihrer ,Gesundheit” bewusst geworden?") oder über Kategorien von Situationen (z.B.: „Wann ist für Sie ,Gesundheit' wichtig?") zu berichten. Mit Hilfe episodischer Interviews wurden beispielsweise Studien durchgeführt, die Zusammenhänge von kritischen Lebensereignissen und dem Auftreten spezifischer Erkrankungen aufdecken konnten.

Grundlage für diese Art der Befragung ist ein Leitfaden, der die anzusprechenden Themen enthält. Sowohl die Auswahl als auch die Gewichtung der Themen wird jedoch den Befragten überlassen. Das Interview wird entweder aufgezeichnet oder in anderer Form protokolliert.

\section{Fokussierte Interviews}

Fokussierte Interviews haben vor allem das Ziel, Hypothesen zu testen. Grundlage ist wiederum ein Leitfaden. Er dient hier dazu, die für die Befragten bedeutsamen Aspekte eines Themas zu erfassen und ihre Reaktionen festzuhalten. Bei Fragen nach dem Gesundheitssystem kann dies z.B. bedeuten, dass Befragte erklären, was für sie zum Gesundheitssystem gehört. Aus den gewonnenen Informationen wird schließlich eine inhaltliche Synthese gebildet, die dann als Grundlage für die weitere Hypothesenbildung dient. Bei fokussierten Interviews gehört es zu den Aufgaben der Interviewerlnnen, Fragestimuli zu setzen und die Befragten ggf. aufzufordern, ihre Aussagen zu präzisieren.

\section{Fokusgruppeninterviews}

In Fokusgruppeninterviews werden Gruppen von Personen zu einer vorher festgelegten Thematik befragt. Die Interviewer geben dabei das Thema vor und strukturieren die daraus entstehende Diskussion. Im Idealfall sollte die Gruppengröße bei acht bis 10 Teilnehmerlnnen liegen. Solche Fokusgruppeninterviews können z. B. dazu dienen, die Bewohner eines Quartiers zu den gesundheitlichen Risiken und Ressourcen in ihrem Wohnumfeld zu befragen. Um einen geeigneten Kreis von Interview-Teilnehmerlnnen zu gewinnen, kann es erforderlich sein, mit potentiellen Teilnehmerlnnen Vorinterviews zu führen. Die Gruppeninterviews sollten auf Band aufgenommen und später in zwei Stufen aufbereitet werden. In einem ersten Schritt werden die Gespräche und Diskussionen mit Hilfe inhaltsanalytischer Techniken ausgewertet. Hierbei werden die Informationen unter Verwendung von zuvor festgelegten Interpretationsregeln und anhand von eigens erstellten Kategoriensystemen klassifiziert. In einem zweiten Schritt werden Diskussionsmuster herausgearbeitet, um z.B. Verzerrungseffekte durch Meinungsführer zu erkennen.

Bei der Interpretation der Daten muss immer berücksichtigt werden, dass es sich bei einem Fokusgruppeninterview um eine künstliche Situation handelt. Die Teilnehmerlnnen wurden durch das Forschungsteam ausgewählt. Die beobachteten und registrierten 
Interaktionen müssen daher nicht den verbalen Reaktionen entsprechen, die in einem natürlichen Rahmen auftreten würden, sodass Übertragungen auf andere Umgebungsbedingungen mit Vorsicht durchgeführt werden müssen. Fokusgruppeninterviews können nicht nur als eigenständige Methode, sondern z.B. auch als Ergänzung zu Fragebogeninterviews eingesetzt werden. Sie können hier u.a. zu einem detaillierteren Verständnis der mit Hilfe von geschlossenen Antwortvorgaben erhobenen Informationen führen.

\section{Einzelfallstudien}

Einzelfallstudien betrachten einen spezifischen Fall im Quer- oder im Längsschnitt. Voraussetzung hierfür ist die Annahme, dass der für die Untersuchung gewählte Fall in seinen relevanten Merkmalen typisch und damit auf andere Fälle übertragbar ist.

Als Untersuchungsobjekte kommen neben Personen auch Gruppen, Institutionen oder Organisationsstrukturen in Frage. Beispiele hierfür wären etwa Schulen, Gewerkschaften oder GesundheitspolitikerInnen. Im Rahmen einer Einzelfallstudie werden dann unterschiedliche Arten von Daten mit dem Ziel gesammelt, im Hinblick auf die Fragestellung ein möglichst vollständiges Bild zu erhalten. So können etwa die Auswirkungen der Einführung von Fallpauschalen auf die alltäglichen Abläufe in einem Krankenhaus anhand einer kleinen Zahl solcher Einrichtungen untersucht werden. Dazu werden z. B. Daten aus den Patientenakten, die Verweildauern und andere routinemäBig erstellte Dokumente herangezogen. Betrachtet werden aber auch typische Interaktionsmuster innerhalb des Krankenhauses sowie Veränderungen bei den alltäglichen Handlungsabfolgen.

Fallstudien können dazu dienen, die Ergebnisse quantitativer Studien zu ergänzen, Hypothesen zu formulieren oder relevante Aspekte einer gegebenen Fragestellung möglichst vollständig auszuleuchten. Die Erkenntnisse aus einer oder wenigen Fallstudien lassen sich jedoch nicht generalisieren, sie können lediglich Unterschiede zwischen den gewählten Untersuchungseinheiten (z. B. Krankenhäusern, Gesundheitssystemen oder einmaligen Ereignissen) aufzeigen.

\section{Internet-Ressourcen}

Auf unserer Lehrbuch-Homepage (www.public-health-kompakt.de) finden Sie Hinweise auf die im Text verwendeten Literaturquellen sowie weiterführende Literatur. 